\title{
A comparative study on characteristics of nanocellulose reinforced thermoplastic starch biofilms prepared with different techniques
}

\author{
Samaneh Karimi, Paridah Md. Tahir, Alain Dufresne, Ali Karimi, and Ali Abdulkhani
}

\begin{abstract}
KEYWORDS: Biofilm, Thermoplastic starch (TPS), Cellulose nanofiber (CNF), Casting technique, Nanocomposite

SUMMARY: Thermoplastic starch (TPS) and cellulose nanofibers (CNFs) obtained from kenaf bast were used to produce biofilms. CNFs were isolated by mechanical defibrillation of purified fibers. Three different casting techniques were chosen for material processing while material ratio, processing time and temperature were kept constant. The effects of casting technique on the morphology, mechanical properties and water sensitivity of the films were investigated by means of field emission scanning electron microscopy (FESEM), tensile and water uptake experiments. Obtained results indicated interesting differences in properties of biofilms and highlighted the importance of casting technique on the final performance of materials.
\end{abstract}

\section{ADRESSES OF THE AUTHURS:}

Samaneh Karimi (samanehkarimi82@gmail.com), Institute of Tropical Forestry and Forest Products (INTROP), University Putra Malaysia.

Paridah Md. Tahir (parida.introp@gmail.com), Institute of Tropical Forestry and Forest Products (INTROP), University Putra Malaysia.

Alain Dufresne (alain.dufresne@ pagora.grenoble-inp.fr), Grenoble Institute of Technology, The International School of Paper, Print Media and Biomaterials (PAGORA),

Ali Karimi (alikarimi@putra.upm.edu.my), Institute of Tropical Forestry and Forest Products (INTROP), University Putra Malaysia. Department of Wood and Paper Science and Technology, Faculty of Natural Resources, University of Tehran.

Ali Abdulkhani (abdolkhani@ut.ac.ir), Department of Wood and Paper Science and Technology, Faculty of Natural Resources, University of Tehran.

Corresponding author: Alain Dufresne

Ascending rate in the price of crude oil and descending rate in the extraction of petroleum in conjunction with global environmental awareness are primary driving forces behind development of the next generation of materials that are compatible with the environment and independent of fossil fuels. Renewable biomaterials have vast potentials to be exploited and are considered as promising candidates to replace petroleum based materials in a sustainable manner (Bondeson, Oksman 2007; Petersson et al. 2007).

Many researchers around the world are deconstructing cellulosic fibers into their elementary building blocks known as cellulose nanofibers (CNFs) or microfibrillated cellulose (MFC), cellulose nanocrystals (CNCs) or whiskers, while one of the promising application is in the composite industry (Hubbe et al. 2008; Siqueira et al. 2010). Incorporation of nanocellulose into polymer matrices for nanocomposite production has been opened an exciting ground and attracted a great deal of attention. Cellulose is famous for being the most abundant organic polymer on earth (Klemm et al. 2011) and is a renewable, biodegradable, sustainable, carbon-neutral and cheap biological raw material. Wood and natural fibers are made of cellulosic nanofibers which possess great mechanical properties and are able to significantly reinforce and enhance properties of polymeric matrices, even at very low volume fractions of the reinforcing phase (Satyanarayana et al. 2009). It is worth to note that the theoretical value of Young's modulus of pure crystalline cellulose domain is about $150 \mathrm{GPa}$ which, for comparison, approaches the value of steel, $200 \mathrm{GPa}$ (Cao et al. 2011) for a much lower density.

Starch is a natural, renewable, and biodegradable polymer produced by many plants as a source of stored energy (Carvalho 2008). It is the second most abundant biomass material in nature (Le Corre et al. 2010). Starches are now considered as the most promising material for the production of biodegradable plastics (PM et al. 2012). Thermoplastic starch (TPS) films can be easily made by a process called gelatinization in the presence of heat, moisture, and other plasticizers (Mathew, Dufresne 2002; Ma et al. 2007; PM et al. 2012). An interesting possibility to improve starch properties is to produce bioinspired nanocomposites through reinforcement of plasticized starch by cellulose nanofibers (Svagan et al. 2007). Previous studies on glycerol plasticized starch materials, reinforced by cellulose nanofibers have shown favourable interactions between cellulose and the polymer matrix (Dufresne et al. 2000; López-Rubio et al. 2007; Svagan et al. 2009). Solution casting technique was predominantly used for processing starch based nanocomposites. However there are limited studies available on the effect of casting techniques on the performance of fabricated materials. The main goal of the current research was to create all green nanocomposite films from kenaf bast nanofibers and starch powder with different casting techniques to study its effect on the final performance of the fabricated biofilms. It is worth to note that a very low content of CNFs has been used in this study. It is very important to evaluate the effect of minimum CNF loading on properties of fabricated films. We believe such loading could be sufficient enough to compensate low mechanical properties of TPS for variety of applications e.g. packaging materials. 


\section{Materials and Methods}

Water retted kenaf (Hibiscus cannabinus v36) bast fibers were kindly supplied by the Institute of Tropical Forestry and Forest Products (INTROP, UPM, Malaysia). Waxy maize starch (amylose content is lower than 1\%) was kindly supplied by Cargill (Krefeld, Germany). All other used chemicals were purchased from Merck Chemicals (Kuala Lumpur, Malaysia).

\section{Nanofiber preparation and characterization}

The preparation and characterization of cellulosic nanofibers extracted from raw kenaf bast fibers were described elsewhere (Karimi et al. 2013). Briefly, kenaf bast fibers underwent an alkali pulping process; afterward a three step bleaching process was applied to eliminate the remaining lignin and hemicellulose. The bleached pulp fiber was then defibrillated to nanoscale cellulosic fibers by mechanical shearing using supermasscolloider MKCA 6-3 from Masuko (Japan).

The structure and size of the obtained nanofibers was studied by transmission electron microscopy (TEM) on a Hitachi model H-7100. A drop of the diluted CNF suspension was deposited on a carbon-coated grid. To enhance the contrast, sample was stained using $2 \mathrm{wt} \%$ uranyl acetate solution and allowed to dry at room temperature. Measurements of dimensions on obtained micrographs were done using the attached image analyzer software. At least fifty measurements were done. The result was reported as the mean value of the data.

$\mathrm{X}$-Ray diffraction measurement was conducted using X'pert Pro Panalytical PW 3040 MPD diffractometer. $2 \theta$ range was between $4^{\circ}$ and $60^{\circ}$. Crystallinity of sample was determined and the crystallinity index (CrI) was calculated by an empirical method using the Eq 1 (Segal et al. 1959):

$$
\mathrm{CrI} \%=\left(\left(I_{002}-I_{a m}\right) / I_{002}\right) \times 100
$$

Where $I_{002}$ is the maximum intensity of the (002) lattice diffraction peak and $I_{a m}$ is the intensity scattered by the amorphous part of the sample.

Characteristics of obtained nanofibers are reported in Table 1.

\section{Film processing}

The starting materials were starch, glycerol (98\% purity), distilled water, and CNF suspension. The glycerol and fiber content in the composition of all films were fixed at
$33.33 \mathrm{wt} \%$ and $0.5 \mathrm{wt} \%$ of total dry weight of materials. For each gram of starch $18.5 \mathrm{~g}$ of distilled water was added.

In the first technique (T1) starch granules were gelatinized upon addition of water and glycerol. Then CNFs were added to interact harmoniously with the prepared matrix. In the second technique (T2) starch granules were first dispersed in the suspension containing the reinforcement and then gelatinized before the glycerol was added. The starch matrix was thereby allowed to interact with the reinforcement prior to addition of plasticizer and hinder the accumulation of plasticizer in the fiber-starch interface. While in the third technique (T3) all starting materials were mixed and processed at the same time in order to obtain a homogeneous dispersion.

The ensuing mixtures were heated on a hotplate (yellow MAG HS7) under both magnetic and mechanical stirring. All ensuing mixtures were sonicated for 30 seconds (amplitude $100 \%$, cycle 0.8) under a UP400S ultrasonic processor, then casted in petri dishes and stored in a ventilated oven at $50^{\circ} \mathrm{C}$ for 48 hours until complete evaporation. The resulting films were kept in a conditioning desiccator of $43 \%$ relative humidity $(\mathrm{RH})$ for 10 days at room temperature and were weighted regularly to ensure the equilibration of the water content in the films before various characterizations. The average formed film thickness was $0.4 \mathrm{~mm}$.

\section{Electron microscopy}

The fractured surface of TPS and nanocomposite strips was examined using Field emission scanning electron microscope (FESEM). Samples were cooled in liquid nitrogen, and then broken. The fractured surface was sputter coated with gold and observed with an accelerating voltage of $5 \mathrm{kV}$.

\section{Tensile tests}

The tensile mechanical properties were investigated using a universal testing machine (Instron 5560). Dumbbell shaped specimens of $4 \mathrm{~mm}$ wide, $30 \mathrm{~mm}$ long and about $0.4 \mathrm{~mm}$ thick were used.

The initial gap between jaws was adjusted to $40 \mathrm{~mm}$. Tests were run at room temperature according to ASTM D882. A crosshead speed of $50 \mathrm{~mm} / \mathrm{min}$ and load cell of $50 \mathrm{~N}$ was used for this test. The average value of five replicates for each sample was taken.

Table 1 - Some characteristics of isolated CNFs

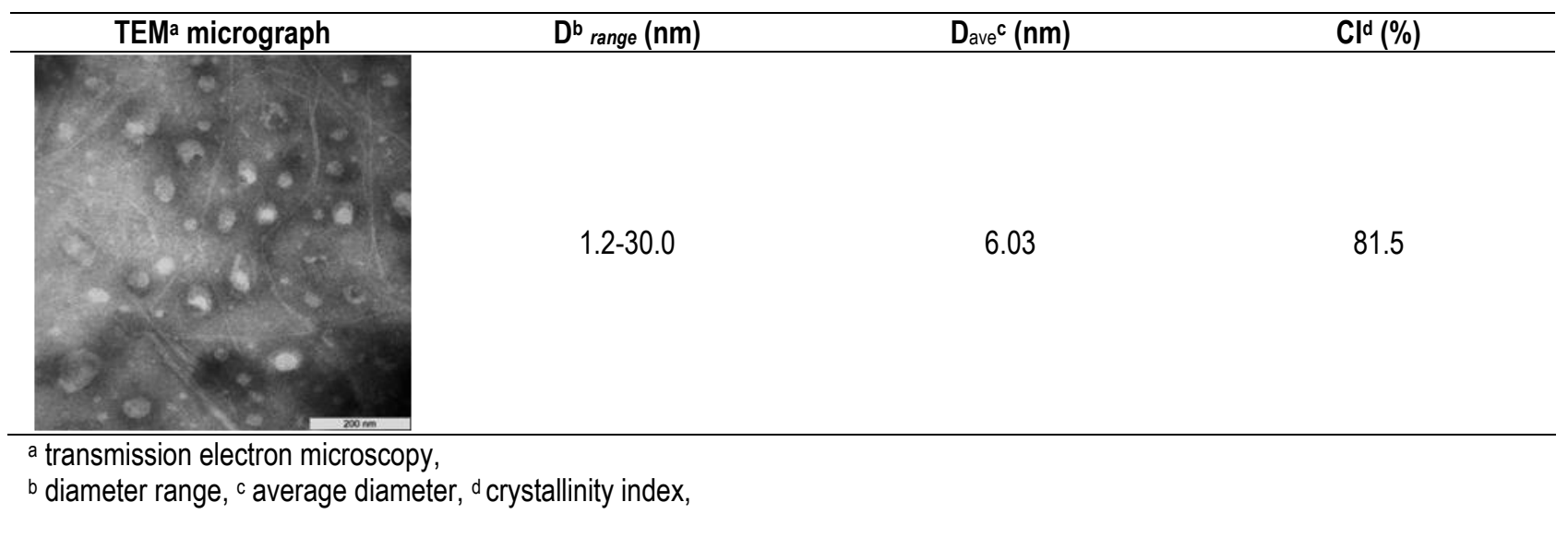




\section{Water uptake}

The kinetics of water absorption was determined for samples according to ASTM D 5229 at $98 \%$ RH. The samples used were thin rectangular strips with dimensions of $50 \times 5 \times 0.4 \mathrm{~mm}^{3}$. The samples were dried at $100^{\circ} \mathrm{C}$ overnight. After weighing, they were conditioned at room temperature in a desiccator of $98 \%$ $\mathrm{RH}$ ( $\mathrm{CuSO} \mathrm{S}_{4} .5 \mathrm{H}_{2} \mathrm{O}$ saturated solution). The conditioning of samples in a high-moisture atmosphere was preferred to the classical technique of immersion in water because starch is very sensitive to water and can partially dissolve after a long exposure to water (Angles, Dufresne 2000). The samples were removed at specific intervals and were weighed until the equilibrium state was reached. Experiments were done in four replicates and the average value was used.

The water uptake $(W U)$ of the samples was calculated according to $E q$ 2:

$$
W U \%=\left(\left(M_{t}-M_{0}\right) / M_{0}\right) \times 100
$$

Where $M_{0}$ and $M_{t}$ are the weights of the sample before exposure to $98 \% \mathrm{RH}$ and after $t \mathrm{~h}$ of exposure to $98 \%$ $\mathrm{RH}$, respectively.

\section{Results and Discussion}

\section{Morphology}

Fig 1 shows the FESEM micrographs of the fractured surfaces of neat TPS as well as biofilms prepared with different techniques.

Due to the small load $\mathrm{f}$ CNFs, they were not easily visible in the micrographs, however no large aggregates were visible and a homogeneous distribution of the CNFs in the TPS matrix was implied. Particularly in the case of film prepared with T3 technique, the good interaction between the plasticizer and starch contributed to the continuous surface of the matrix.
Such an even and uniform distribution of the filler in the matrix could play an important role in improving the mechanical performance of the resulting nanocomposite films (Savadekar and Mhaske 2012).

\section{The mechanical properties}

The mechanical properties of the neat TPS and the nanocomposite films reinforced with $0.5 \mathrm{wt} \% \mathrm{CNFs}$ processed with different techniques were investigated by tensile testing at room temperature. The results of mechanical properties are shown in Fig 2. Tensile strength, elongation at break and Young's Modulus were evaluated from the experimental stress-strain curves. Comparing tensile strength of fabricated materials, it is obvious that despite very low volume fraction of CNFs, the tensile strength was increased for all three nanoreinforced biofilms with respect to the blank sample (neat TPS).

This can be explained by the reinforcement ability of the homogeneously dispersed high-performance CNFs fillers in the TPS matrix and the strong hydrogen bonding interaction between CNFs and TPS molecules (Soykeabkaew et al. 2004; Liu et al. 2006; Soykeabkaew et al. 2012). As expected, the elongation at break reduced with the addition of cellulose nanofibers.

The modulus increased from 40.9 $\mathrm{MPa}$ to $53.6 \mathrm{MPa}$ $(31 \%)$ by changing the processing technique from $\mathrm{T} 1$ to T3. This is attributed to better interfacial interactions and hydrogen bonding between CNFs and starch molecules using this technique. Also according to FESEM micrographs (Fig 1), the film prepared with this technique showed a smoother and more homogenous surface.

A significant improvement in mechanical properties was reported for biofilm prepared with T3 technique.

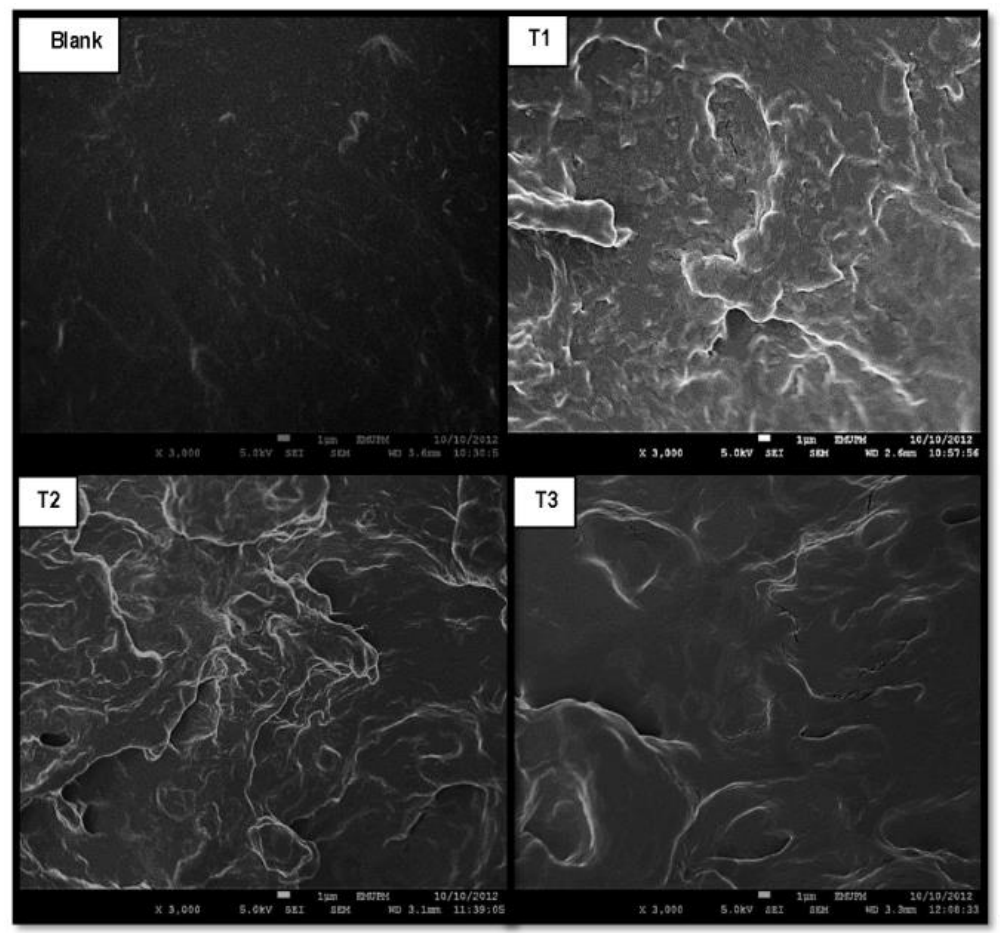

Fig 1 - FESEM micrographs of the blank sample (TPS) and nanocomposite films prepared with different techniques (T1, T2 and T3) 


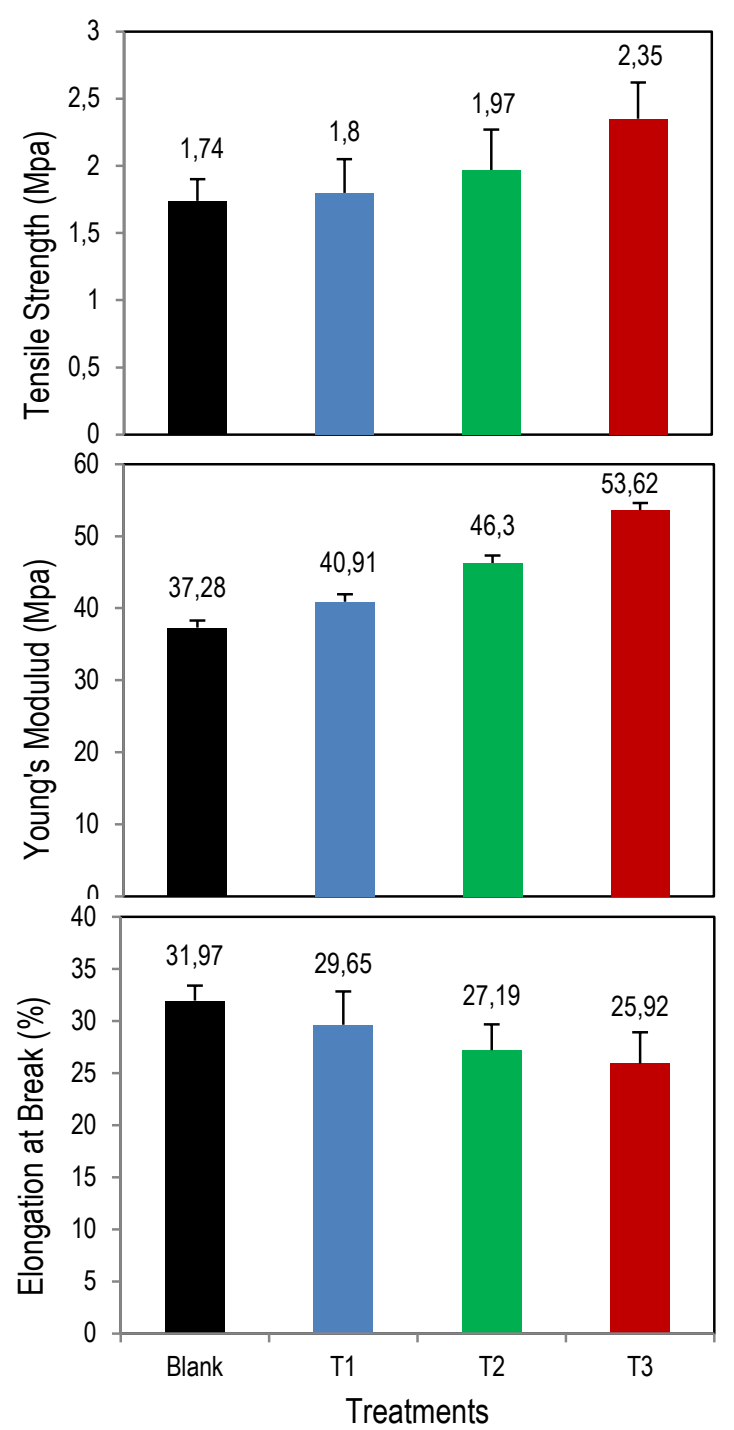

Fig 2 - Tensile strength (MPa), Young's Modulus (MPa) and Elongation at break (\%) of fabricated materials with varying casting techniques (CNF load $=0.5 \mathrm{wt} \%$ )

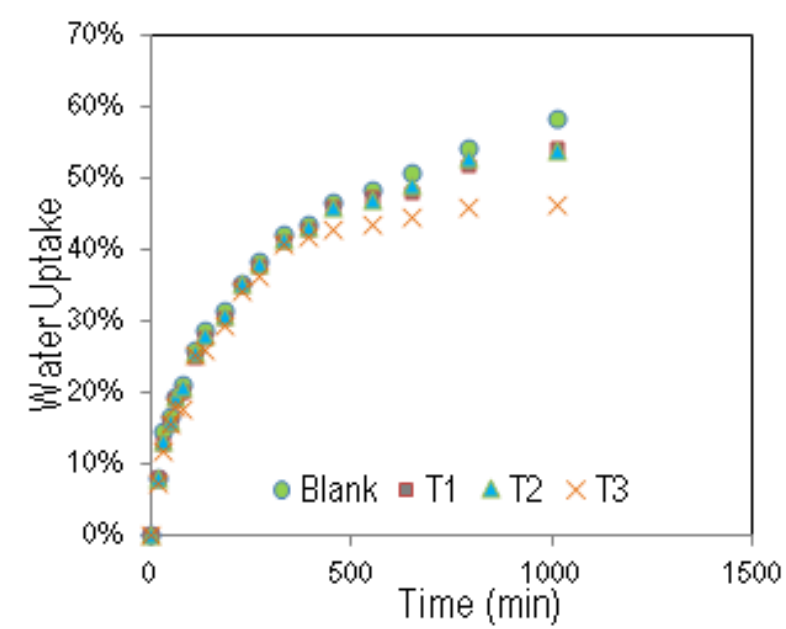

Fig 3 - Water uptake of biofilms conditioned at $98 \% \mathrm{RH}$ as a function of time (sample codes are indicated in the figure)

\section{Water uptake}

The water uptake of the fabricated materials as a function of time is shown in Fig 3. After incorporating CNFs into the TPS matrix, the water uptake was decreased. Therefore, it can be concluded that the swelling of the material is hindered in the presence of very low volumes of CNFs within the TPS matrix. This phenomenon can be ascribed firstly to the lower water uptake of CNFs compared to thermoplastic starch; and secondly to the presence of strong filler/filler and matrix/filler hydrogen bonding interactions.

As can be seen from Fig 3, no significant improvement in water sensitivity of biofilms prepared with T1 and T2 techniques were observed. However, the trend was different in the case of T3 casted film and a significant reduction in water uptake of this film was reported. This observation was in correlation with obtained data on morphology and tensile strength for T3 casted film

\section{Conclusion}

Cellulose nanofibers from kenaf bast were isolated and used to reinforce a TPS matrix. To understand the effect of casting technique on characteristics of obtained nanocomposites, three techniques (coded as $\mathrm{T} 1, \mathrm{~T} 2$ and $\mathrm{T} 3$ ) were used in processing of materials. FESEM micrographs of the cryo-fractured surface of fabricated materials displayed a homogeneous dispersion of CNFs within the TPS matrix. However, the casted film with the T3 technique showed the most homogeneous surface over all. With addition of only $0.5 \mathrm{wt} \%$ of CNFs tensile strength and Young's modulus of T3 film increased significantly by $35 \%$ and $44 \%$, respectively, compared to the blank sample and the elongation at break decreased by $19 \%$. Addition of the nanoreinforcement decreased the water sensitivity of the TPS film. Significant improvement was reported for the T3 biofilm as $12 \%$ decrease in water content at equilibrium was observed. Finally, all obtained results were correlated and demonstrated the reinforcement ability of CNFs even in very low fraction from one side, and better compatibility and performance of biofilms prepared with $\mathrm{T} 3$ technique, from the other side. The hypothesis would be in the $\mathrm{T} 3$ technique the nanocomposite processing is mostly based on starchcellulose interactions compared to other treatments, where hydroxyl carbohydrate sites seems saturated with glycerol molecules. In the latter case, the transfer at the interface is weaker during mechanical solicitations in agreement with the obtained results.

\footnotetext{
Acknowledgements

Authors would like to thank Dr. Hanieh Kargarzadeh for her sincere assistance in performing tensile mechanical tests. Many thanks are also expressed to Mrs. Ashraf Zohrabi for her faithful support and assistance in preparing nanocomposite films. The institute of tropical forestry and forest products (INTROP) is fully acknowledged for financial and technical support of the project.
} 


\section{Literature}

Angles, M. N. and A. Dufresne (2000): Plasticized starch/tunicin whiskers nanocomposites. 1. Structural analysis, Macromolecules, 33(22), 8344-8353.

Bondeson, D. and K. Oksman (2007): Dispersion and characteristics of surfactant modified cellulose whiskers nanocomposites, Composite Interfaces, 14, 7(9), 617-630.

Cao, X., Y. Habibi, W.L.E. Magalhães, O. Rojas and L.A. Lucia (2011): Cellulose nanocrystals-based nanocomposites: Fruits of a novel biomass research and teaching platform, Current Science, 100(8), 1172-1176.

Carvalho, A. J. (2008): Starch: major sources, properties and applications as thermoplastic materials. In Monomers, polymers and composites from renewable resources, M.N. Belgacem and A. Gandini (eds.), Elsevier, Oxford, pp. 321342.

Dufresne, A., D. Dupeyre, and M.R. Vignon (2000): Cellulose microfibrils from potato tuber cells: processing and characterization of starch-cellulose microfibril composites, Journal of Applied Polymer Science, 76(14), 2080-2092.

Hubbe, M. A., O. J. Rojas, L.A. Lucia and M. Sain (2008): Cellulosic nanocomposites: a review, BioResources, 3(3), 929-980.

A. Karimi, A. Dufresne and A. Abdulkhani (2013): Kenaf bast cellulosic fibers hierarchy: A comprehensive approach from micro to nano accepted in Carbohydrate Polymers.

Klemm, D., F. Kramer, S. Moritz, T. Lindström, M. Ankerfors, D. Gray and A. Dorris (2011): Nanocelluloses: A New Family of Nature-Based Materials, Angewandte Chemie International Edition, 50(24), 5438-5466.

Le Corre, D., J. Bras, and A. Dufresne (2010): Starch nanoparticles: a review, Biomacromolecules, 11(5), 11391153.

Liu, H., L. Yu, F. Xie and L. Chen (2006): Gelatinization of cornstarch with different amylose/amylopectin content, Carbohydrate Polymers, 65(3), 357-363.

López-Rubio, A., J. Lagaron, M. Ankerfors, T. Lindström, D. Nordqvist, A. Mattozzi and M.S. Hedenqvist (2007): Enhanced film forming and film properties of amylopectin using micro-fibrillated cellulose, Carbohydrate Polymers, 68(4), 718-727.

Ma, X., J. Yu, and N. Wang (2007): Production of Thermoplastic Starch/MMT-Sorbitol Nanocomposites by Dual-Melt Extrusion Processing, Macromolecular Materials and Engineering, 292(6), 723-728.

Mathew, A. P. and A. Dufresne (2002): Plasticized waxy maize starch: effect of polyols and relative humidity on material properties, Biomacromolecules, 3(5), 1101-1108.

Petersson, L., I. Kvien, and K. Oksman (2007): Structure and thermal properties of poly (lactic acid)/cellulose whiskers nanocomposite materials, Composites science and technology, 67(11-12), 2535-2544.

PM, V., A. P. Mathew, et al. (2012): Starch-based bionanocomposites: Processing and properties. In Polysaccharide Building Blocks: A Sustainable Approach to the Development of Renewable Biomaterials, Y. Habibi and L.A. Lucia (eds.), Wiley, Hoboken, New Jersay, pp. 287-306
Satyanarayana, K. G., G. G. Arizaga, and F. Wypych (2009): Biodegradable composites based on lignocellulosic fibers-an overview, Progress in Polymer Science, 34(9), 982-1021.

Savadekar, N. and S. Mhaske (2012): Synthesis of nano cellulose fibers and effect on thermoplastics starch based films, Carbohydrate Polymers, 89(1), 146-151.

Segal, L., J. Creely, A.E. Martin Jr, and C.M. Conrad (1959): An empirical method for estimating the degree of crystallinity of native cellulose using the X-ray diffractometer, Textile Research Journal, 29(10), 786-794.

Siqueira, G., J. Bras, and A. Dufresne (2010): Cellulosic bionanocomposites: a review of preparation, properties and applications, Polymers, 2(4), 728-765.

Soykeabkaew, N., N. Laosat, A. Ngaokla, N. Yodsuwan and T. Tunkasiri (2012): Reinforcing potential of micro-and nano-sized fibers in the starch-based biocomposites, Composites science and technology, 72(7), 845-852.

Soykeabkaew, N., P. Supaphol, and R. Rujiravanit. (2004): Preparation and characterization of jute-and flax-reinforced starch-based composite foams, Carbohydrate Polymers, 58(1), 53-63.

Svagan, A. J., M. A. Azizi Samir, and L.A. Berglund (2007): Biomimetic polysaccharide nanocomposites of high cellulose content and high toughness, Biomacromolecules, 8(8), 2556-2563.

Svagan, A. J., M. S. Hedenqvist, and L. Berglund (2009): Reduced water vapour sorption in cellulose nanocomposites with starch matrix, Composites science and technology, 69(3), 500-506

Manuscript received October 13, 2013 Accepted January 14, 2014 